\title{
Study on FSI Analysis Method of a Large Hydropower House and Its Vortex-Induced Vibration Regularities
}

\author{
Liaojun Zhang, ${ }^{1}$ Guojiang Yin $\mathbb{D}^{1},{ }^{1}$ Shuo Wang $\mathbb{D}^{1},{ }^{1}$ and Chaonian Guan ${ }^{1,2}$ \\ ${ }^{1}$ College of Water Conservancy and Hydropower Engineering, Hohai University, Nanjing 210098, China \\ ${ }^{2}$ Power China Zhongnan Engineering Corporation Limited, Changsha 410014, China \\ Correspondence should be addressed to Guojiang Yin; gjyin@hhu.edu.cn and Shuo Wang; qqwang@hhu.edu.cn
}

Received 21 October 2019; Revised 24 September 2020; Accepted 15 October 2020; Published 28 October 2020

Academic Editor: Hui Yao

Copyright $(92020$ Liaojun Zhang et al. This is an open access article distributed under the Creative Commons Attribution License, which permits unrestricted use, distribution, and reproduction in any medium, provided the original work is properly cited.

\begin{abstract}
The working principle of a large hydropower station is to guide the high-pressure water flow to impact the turbine to rotate and generate electricity. The high-pressure water flow impacts the turbine blades, which forms complex high-speed eddy currents in the spiral case and the draft tube and causes complicated vortex-induced vibration problems. Traditionally used harmonic response methods and dynamic time-history analysis methods are difficult to reflect this complex fluid-solid dynamic coupling problem. In this paper, the bidirectional fluid-structure interaction (FSI) simulation analysis theory for a large hydropower house is studied, and the analysis methods of geometric simulation, mechanical simulation, and vibration energy transmission path simulation are presented. A large-scale 3D fluid-hydraulic machinery-concrete structure coupled model of a hydropower house is established to study the vortex-induced vibration mechanism and coupled vibration law during transient unit operation. A comparison of the fluid results against the in-site data shows good agreement. Structural responses of vibration displacement, velocity, and acceleration reveal coupled regularity of hydraulic machinery-concrete structure-fluid during blades rotating periods, and it comes to the conclusion that the turbine blade rotation is the main vibration source of the hydropower house. The research results can provide a scientific basis for the design and safe operation of the hydropower house.
\end{abstract}

\section{Introduction}

The vibration problem during the operation of the hydropower station is one of the key issues in the design of the hydropower house, which mainly involves mechanical vibration induced by the operation of the hydraulic turbine, hydraulic vibration induced by the coupled interaction between the high-speed rotating flow and the structure, and concrete structural vibration through the transmission of the vibration source. With the increase of unit capacity and structural dimensions, strong vibrations may occur and affect the stable operation of the hydropower station $[1,2]$. Relevant cases can be referred to Yantan and Xiaolangdi hydropower stations in China, and Grand Coulee Project in the United States. Obvious vibrations have also been detected during the start-up and shutdown processes of Xiangjiaba hydropower house, which owns currently the largest unit capacity of $800 \mathrm{MW}$ in China. In order to ensure the stable operation of the entire hydropower station and provide practical design and construction reference, it is of great significance for the vibration control and safe operation of the hydropower house to study the fundamental theory reflecting fluid-solid coupling features, to understand the rules of hydraulic pulsation, structural vibration, and their dynamic interaction in the entire hydropower house, as well as finding out the vibration source of the hydropower house.

Computational fluid dynamics (CFD) is considered a powerful method to provide insight into the flow features in hydropower components. Numerous simulations have been performed for the analysis of the fluid flow in both hydraulic turbines and pumps [3]. Li et al. [4] conducted CFD analysis of the pressure pulsations in a prototype pump turbine in turbine mode with different blades numbers. Kc et al. [5] investigated transient flow features inside a Francis turbine including the distribution of pressure, streamline, and vortex 
rope at full-load and part-load operation. Minakov et al. [6] presented numerical simulation results like vortex rope and pressure pulsation for two Francis turbines at different openings of the wicket gate. Wei et al. [7] performed a study on features of flow, performance, cavitation in the runner and vortex rope, and pressure fluctuations in the draft tube of the perforated and imperforated turbines. Trivedi et al. [8] focused on the experimental and numerical studies of the runaway condition and presented the flow field and its effects on the runner blades. Chalghoum et al. [9] analyzed the effect of the impeller diameter and number of blades on the pressure evolution in a centrifugal pump during the starting process. Li et al. [10] studied the hump characteristics of a pump turbine with the presentation of transient streamline and velocity in the tandem cascade passages at different discharges. In research of hydraulic machinery, a threedimensional CFD model was generally established with the inner characteristics as its main focus, such as variation of the vortex core, regularity of pressure pulsation in time and frequency domain, velocity, and streamline distribution in spatial domain.

Computational structural dynamics (CSD) is one of the most accurate and effective calculation tools for simulating complex structural vibrations. It can reveal the real-time vibration deformation, vibration velocity, and vibration stress of the combined structure under complex loads and help to understand the mechanism of structural vibration damage, which are very important for the vibration control and optimization design of the hydropower house. It is vital to conduct structural vibration analysis of the hydropower house induced by flow excitations during turbine operation. Numerous scholars have conducted relevant research using the quasi-static method, harmonic response method, and the dynamic time-history method $[11,12]$. Zhang and Zhang [13] studied the nonlinear dynamic response of the hydropower house under the frame of isotropic damage coupled with plasticity for concrete and found that tensile damage in the concrete surrounding the spiral case showed little impact on the superstructure but exerted a significant effect on the substructure. The dynamic time-history method was adopted in their study, in which time pressure distribution inside the concrete flow passage was artificially supposed, and the time-varying pressure values were exacted from nine measuring points.

Fluid-structure interaction (FSI) is the state-of-the-art technique for studying fluid and solid dynamic interactions, which combines CFD and CSD theory and has increased applications in complicated hydraulic mechanical engineering. One-way coupled FSI method, in which the steady-state CFD simulation provides the fluid pressure for FEA calculation as a load or a boundary condition, is generally adopted in previous research. Negru et al. [14] and Saeed et al. [15] analyzed the stresses induced in a Francis turbine runner by steady flow for different operating regimes and concluded that the sensitive areas to fatigue crack initiation was in the trailing edge between the blades and the crown. Xiao et al. [16] conducted a one-way FSI analysis of the flow field, the static and dynamic stress in the runner of a Francis turbine, and concluded that the dynamic stress was the main contributor to fatigue and cracks of the blade during off-design operation. Luna-Ramírez et al.
[17] performed a failure analysis of runner blades based on the results of fluid pressure in CFD simulation and concluded that the most likely cause of failure was that turbine operation at low loads, accelerated by detected cavitation, and high water levels in the upper reservoir and tailwater. Although the one-way FSI method could economically describe structural responses under the fluid effect, it is known to be insufficient to evaluate structural integrity due to no feedback of the blade deflection on the flow. Two-way coupled FSI could fill this gap by conducting iterations between fluid and solid systems. Dompierre et al. [18] calculated the dynamic behavior of a Francis turbine runner under operating conditions based on the two-way FSI method introduced by ANSYS. Schmucker et al. [19] utilized two different methods to demonstrate the influence by means of two-way FSI simulation of the propeller turbine on the hydraulic performance. All their research highlights the importance of two-way coupled FSI simulation in the application of hydraulic engineering, but, without taking the concrete structure of the hydropower house into account, their concern is mainly the hydraulic machinery.

Zhang et al. [20] optimized $\mathrm{C}_{2}$ interpolation method for data transfer of the fluid-structure coupling interface in the hydropower house and conducted a vibration analysis of the hydropower house. Most previous research rarely considered dynamic interaction between powerhouse structure and the entire water flow and rarely performed a 3D fully fluidsolid coupling simulation, in which the fluid analysis was conducted with the CFD method, and the entire structure of hydropower house was investigated by the two-way FSI approach. Therefore, it is a tremendous need to apply the FSI method to the hydropower house research for detailed study.

In existing papers for studying the vibration of the hydropower house, the fluid model and hydraulic machinery are often omitted, or the machinery is simulated as a onedimensional numerical model. The main purpose of this paper is to perform the research on the bidirectional FSI simulation analysis theory applicable to large hydropower houses by combining CFD theory and CSD theory and to propose the analytical method of geometric simulation, mechanical simulation, and vibration energy transmission path simulation. CFD based on the finite volume method (FVM) is adopted in the fluid domain to explore hydrodynamic features of the turbulent flow. CSD based on the finite element method (FEM) is utilized in the solid domain to study structural dynamic responses of the hydropower house. A bidirectional iterative FSI method is adopted to solve the dynamic interaction between the fluid and solid domains. The transient structural dynamics model is employed to study the vibration characteristics of the hydropower house structures. Based on the powerful FSI function of the commercial software ADINA, a fluid-hydraulic machinery-concrete structure coupled model for Xiangjiaba hydropower house is established, including the concrete structure of the hydropower house, the mechanical structure of the turbine, the generator, the upper and lower brackets, and the fluid inside the entire flow passage. The techniques of the sliding mesh and the upwind scheme are adopted to carry out the FSI bidirectional iterative calculation, and the high-nonlinear dynamic problems are effectively resolved. The vortex-induced vibration mechanism 
and coupled vibration regularity during transient operation of the unit are discussed. The main vibration source and the transmission path of the hydropower house vibration are clarified, which provides an important reference for the design and construction of the hydropower house.

\section{FSI Simulation Analysis Theory, Model, and Method}

2.1. Basic Equations. In this section, the basic equations for the numerical simulation are introduced.

The basic equation for the turbulence flow is given in the following.

In the fluid domain of the hydropower house, the continuity equation is adopted to satisfy the conservation of the mass, as shown in the following equation:

$$
\frac{\partial\left(\rho u_{i}\right)}{\partial x_{i}}=0,
$$

where $\rho$ is the fluid density and $\mu_{i}$ are the velocity components.

As a built-in feature of the ADINA software, SpalartAllmaras detached eddy simulation (SA-DES) turbulence equation was adopted to model the turbulence and produce the eddy viscosity for the flow. Derived from the SA oneequation eddy-viscosity RANS model, SA-DES combines the advantages of large eddy simulation (LES) and RANS method and could simulate the large-scale turbulent pulsations accompanied by the precession of vortex in hydraulic machinery economically and reliably $[6,21]$. The equation of the SA-DES turbulence model is expressed as follows [22-24]:

$$
\frac{\partial}{\partial t}(\rho \widetilde{v})+\frac{\partial}{\partial x_{j}}\left(\rho \widetilde{v} u_{j}\right)=c_{b 1} \rho \widetilde{S} \widetilde{v}+\frac{1}{\sigma}\left\{\frac{\partial}{\partial x_{j}}\left[\rho(v+\widetilde{v}) \frac{\partial \widetilde{v}}{\partial x_{j}}\right]+c_{b 2} \rho \frac{\partial \widetilde{v}}{\partial x_{j}} \frac{\partial \widetilde{v}}{\partial x_{j}}\right\}-c_{\omega 1} f_{\omega} \rho\left(\frac{\widetilde{v}}{d_{\mathrm{DES}}}\right)^{2}
$$

where $\widetilde{v}$ is the modified turbulent viscosity linked to the turbulent viscosity $v_{t}$ and a wall function $f_{v 1}$ by $\widetilde{v}=v_{t} / f_{v 1}$, $f_{v 1}=\left(\chi^{3} /\left(\chi^{3}+c_{v 1}^{3}\right)\right), \chi=(\widetilde{v} / v)$, and $c_{v 1}=7.1 . v$ is the molecular viscosity. $\widetilde{S}$ is the modified vorticity defined in terms of the magnitude of the vorticity $S$ by $\widetilde{S}=S+\left(\widetilde{v} / k^{2} d^{2}\right) f_{v 2}$, $S=\sqrt{2 S_{i j} S_{i j}}, f_{v 2}=1-\left(\chi /\left(1+\chi f_{v 1}\right)\right)$. Other model coefficients are given by $c_{b 1}=0.1335, c_{b 2}=0.622, \sigma=(2 / 3), c_{\omega 1}=$ $\left(c_{b 1} / k^{2}\right)+\left(\left(1+c_{b 2}\right) / \sigma\right), f_{\omega}=g\left(\left(1+c_{\omega 3}^{6}\right) /\left(g^{6}+c_{\omega 3}^{6}\right)\right)^{(1 / 6)}$, $c_{\omega 3}=2, g=r+c_{\omega 2}\left(r^{6}-r\right), r=\left(\widetilde{v} / \widetilde{S} k^{2} d^{2}\right)$, and $c_{\omega 2}=0.3$.

As the switch function of the LES-SGS and RANS turbulence model, $d_{\mathrm{DES}}$ is described in the form of the following equation:

$$
L^{T} \tau_{s}+\rho_{s} f_{s}=\rho_{s} \ddot{u}
$$

where $d$ is the nearest distance to the wall and $\Delta_{\max }$ is the maximum local grid size, $C_{\mathrm{DES}}=0.65$.

The basic equation for the structural vibration is given in the following.

The control equation for the structure of the hydropower house can be written in the following equation [25]:

$$
L^{T} \tau_{s}+\rho_{s} f_{s}=\rho_{s} \ddot{u}
$$

where $\tau_{s}, f_{s}, \ddot{u}, \rho_{s}$, and $L^{T}$ denote the solid stress tensor, the solid body force, the solid particle acceleration vector, the solid density, and the differential operator, respectively.

\subsection{Numerical Simulation Model}

2.2.1. Mesh Independence Test. Since nodes number greatly affects the simulation accuracy especially in the transient fluid calculation, a mesh independence test has been conducted for the fluid model to obtain a reasonable number of the computational grids.

In the fluid domain, FSI boundary condition was replaced by a no-slip wall boundary to perform the CFD test. With the steady-state analysis, head $H$ in different grid size was evaluated by imposing velocity at the inlet and outflow at the outlet. Figure 1 shows the influence of grid size on the ratio of calculated head $H$ to rated head $H_{0}$. The fluid mesh was required to converge till obtaining the variable residual of $1 e-4$. There was no significant variation for $H / H_{0}$ values with nodes number beyond $C$. Based on comprehensive consideration of the simulation accuracy and intense computational resources required by the two-way iterative FSI solver, nodes number $C$ with 442704 elements and 481906 grid nodes in the fluid domain was selected to perform transient state FSI analysis.

2.2.2. Computational Model. The previously described coupling method is applied to the Xiangjiaba hydropower house owning currently the largest unit capacity of $800 \mathrm{MW}$ in China. In order to represent realistic hydrostructural dynamic features, a more comprehensive model and delicately divided finite element mesh are obtained according to the actual construct of mechanical structures like the hydraulic turbine, generator, and upper and lower brackets.

Figure 2 shows the computational model for fluid and solid domain. As shown in Figure 2(a), an integral 3D fluid-solid coupling model of the hydropower house is established to analyze the coupled vibration of the structure. The model of the entire fluid domain through the turbine is established to obtain the fluid pressure pulsation, depicted in Figure 2(b). Figure 2(c) is the axial section view of the hydropower house for a clearer depiction. The vibration energy transmission path is well simulated, in which fluid pressure pulsation is firstly transmitted from the runner blade to the head cover and also to concrete structure through the bearing and the bracket 


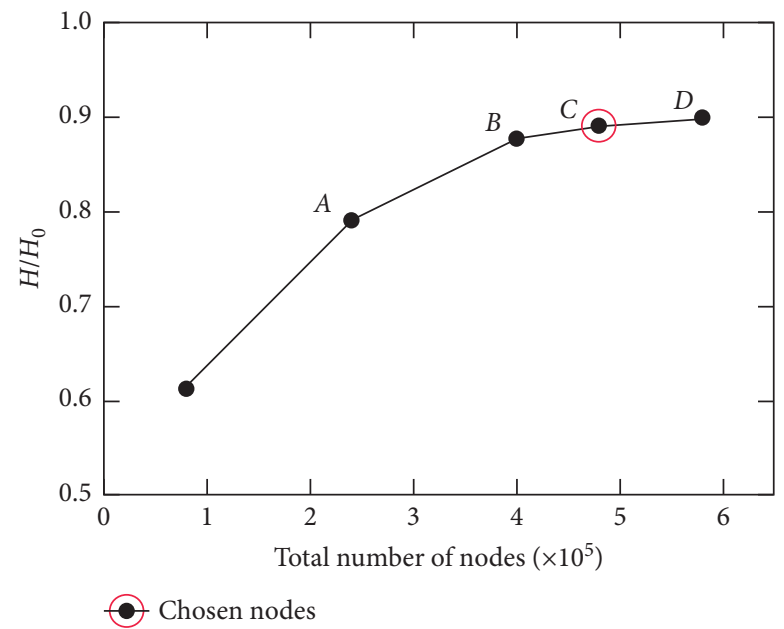

Figure 1: Mesh independence test.

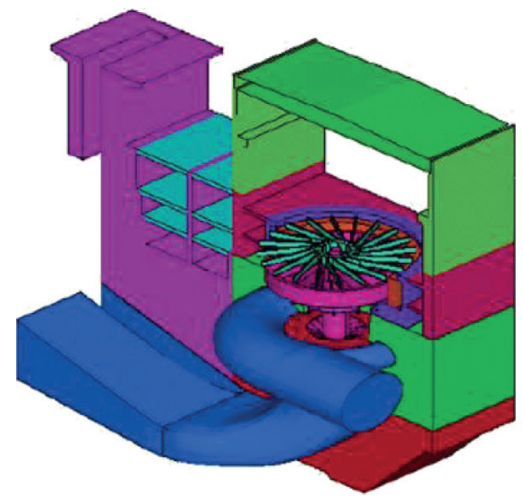

(a)

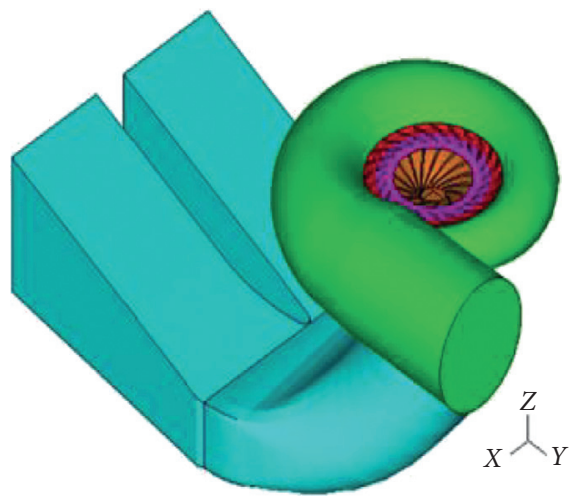

(b)

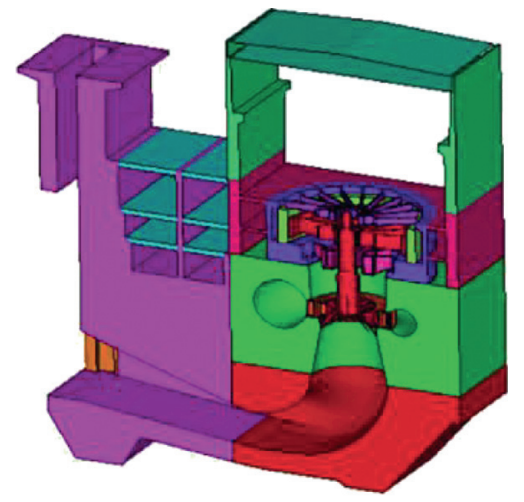

(c)

FIgure 2: Computational domain (Figure 2 is reproduced from Shuo Wang et al., 2019.). (a) FSI model. (b) Fluid model. (c) Solid model in the axial plane.

connected by the principal axis. The FSI coupled model and transmission path simulation could reflect the transient transmission process of the structural vibration induced by unit operation and the vibration transmitting in the entire structure of the hydropower house.

(1) Fluid Model Simulation. Figure 3 illustrates the structured grids for four fluid subregions, involving fluid in a spiral case, fluid around stay vane and guide vane and runner, and fluid in a draft tube. Since the shape and configuration of the flow channel greatly influence internal velocity and hydraulic pressure field, 3D fluid element is chosen in the fluid domain with fine and structured grids designed on basis of different topology. Finer grids are applied to the rotational zone and field near the wall in the fluid domain to ensure high-accuracy results. The fluid domain comprises 442704 3D fluid elements and 481906 grid nodes.

(2) Solid Model (Concrete and Mechanical Structure) Simulation. As the core component of the hydraulic turbine, the runner, motivated by high-speed flowing water, drives the generator rotor through the main shaft and transmits the energy of the vibration source upwards through its coupling with water. Vertical dynamic loads such as runner-axis-rotor coupled gravity and axial water pressure imposed on the runner are transferred to the base pier through thrust bearing and bracket. As the lower bracket, it bears the main vibration load generated during the operation of the unit. The upper bracket is connected to the stator and floor to transmit the load exerted by the weight of its own and other structures. Comprehensive components of concrete and mechanical structure in the hydropower house are modeled to obtain a realistic and accurate simulation of vibration energy transmission path. Figure 4 shows the grids for concrete structure, including turbine floor, dynamo floor, draft tube block structure, sidewall, and roof. The most concrete structure is simulated using a $3 \mathrm{D}$ solid element except a shell element for the roof. 3D solid elements are also adopted for modeling the brackets, beam, and floor to avoid serious stress concentration. Mesh of mechanical structure, including runner, axis and rotor, upper and lower bracket, and stay and guide vane, is presented in Figure 5. The solid 


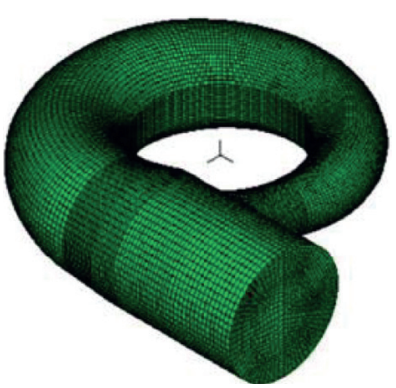

(a)

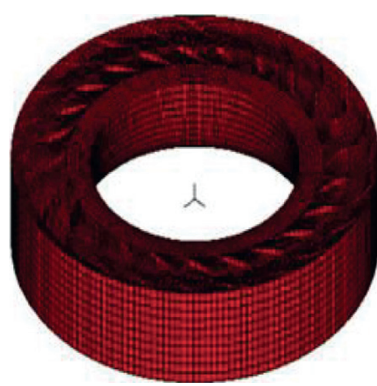

(b)

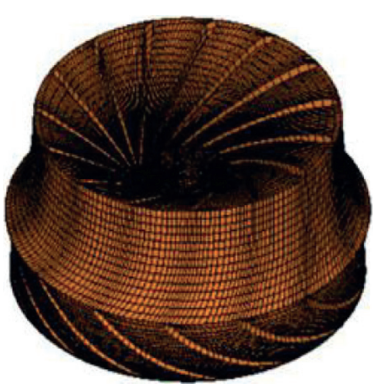

(c)

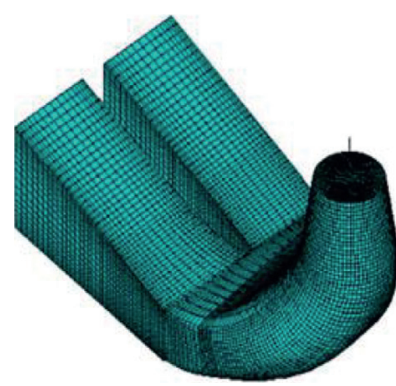

(d)

FIGURE 3: 3D structured grids for the fluid domain (Figure 3 is reproduced from Shuo Wang et al., 2019.). (a) Spiral case. (b) Stay and guide vane. (c) Runner. (d) Draft tube.

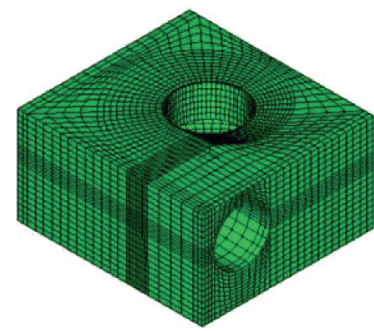

(a)

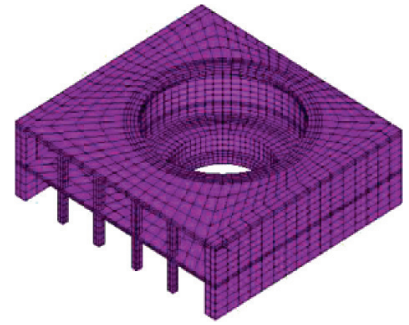

(b)

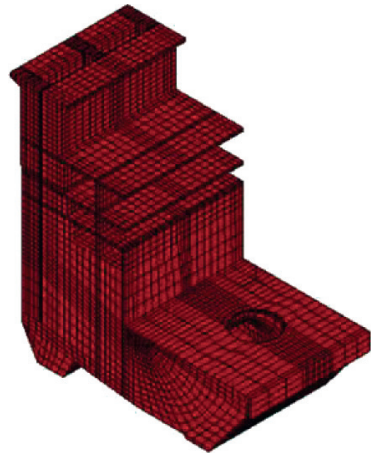

(c)

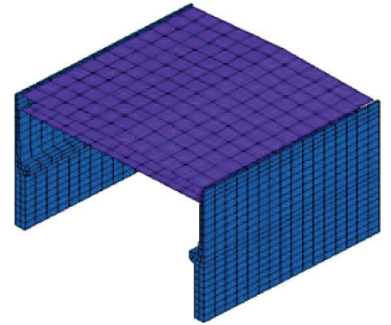

(d)

Figure 4: 3D structured grids for the concrete domain. (a) Turbine floor. (b) Dynamo floor. (c) Draft tube block structure. (d) Sidewall and roof.

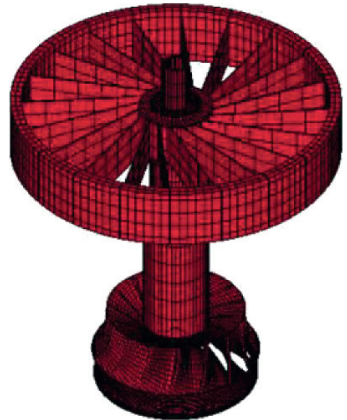

(a)

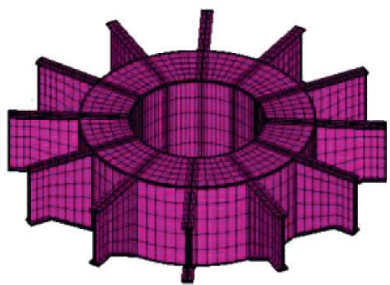

(b)

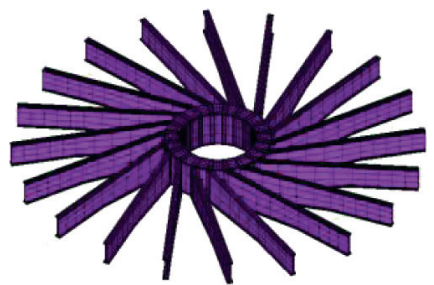

(c)

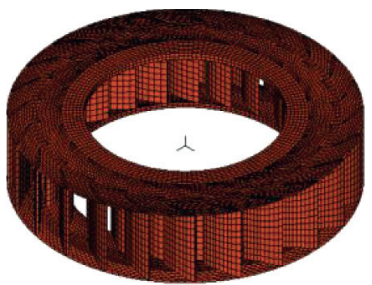

(d)

Figure 5: 3D structured grids for the mechanical domain. (a) Runner, axis, and rotor. (b) Lower brackets. (c) Upper brackets. (d) Stay and guide vane.

domain is composed of 187636 elements and 136752 grid nodes.

Table 1 shows the main computational parameters. In the current research, the turbine operates at best efficiency point (BEP) with a rated head of $100 \mathrm{~m}$ and a rated flow rate of $890 \mathrm{~m}^{3} / \mathrm{s}$. For the fluid domain, density and viscosity are set as $998.2 \mathrm{~kg} / \mathrm{m}^{3}$ and $0.001003 \mathrm{~Pa} \cdot \mathrm{s}$, respectively. The number of runner blades is 15 , and the number of guide vanes is 24 . As for the inlet, a uniform velocity of $7.613 \mathrm{~m} / \mathrm{s}$ is applied in terms of the flow rate of $890 \mathrm{~m}^{3} / \mathrm{s}$. The turbulent flow is supposed to be fully developed at the outlet with outflow set as the outlet boundary condition. The rotational speed of $75 \mathrm{rpm}$ is assigned to the hydraulic turbine.

2.3. Calculation Method. Geometric simulation, mechanical simulation, and transmission path simulation are performed to study the coupled dynamic model. As mentioned above, appropriate element types and well-designed mesh division are chosen to perform geometric and mechanical simulation, 
TABle 1: Computational parameters.

\begin{tabular}{lcccc}
\hline \multirow{2}{*}{ Design parameters of the turbine } & \multicolumn{3}{c}{ Material parameters of the structure } \\
& \multicolumn{4}{c}{ Concrete } \\
\hline Rated head $H(\mathrm{~m})$ & 100 & Density $\rho_{c}\left(\mathrm{~kg} / \mathrm{m}^{3}\right)$ & 2500 & Density $\rho_{s}\left(\mathrm{~kg} / \mathrm{m}^{3}\right)$ \\
Flow rate $Q\left(\mathrm{~m}^{3} / \mathrm{s}\right)$ & 890 & Elastic modulus $E_{c}(\mathrm{GPa})$ & 21 & Elastic modulus $E_{s}(\mathrm{GPa})$ \\
Rated rotation speed $n(\mathrm{rpm})$ & 75 & Poisson ratio $\mu_{c}$ & 0.167 & 210 \\
\hline
\end{tabular}

reflecting realistic coupling features of hydrodynamics in turbulent flow and structural dynamics in hydropower house. Accurately modeling the comprehensive components in the hydropower house well presents the vibration energy transmission path, in which fluid pressure pulsation is generally transmitted to the runner blade and the head cover and then to the concrete structure through the bearing and the bracket connected by the principal axis.

Two-way iterative FSI method is adopted to perform the simultaneous calculation with CFD solver based on FVM in the fluid domain and CSD solver based on FEM in the solid domain. The transient state is set in ADINA CFD module and the dynamics-implicit algorithm is employed in the ADINA structure module. In this solution, the fluid and solid solution variables are fully coupled. The coupling procedure of the two-way iterative FSI method can be summarized as follows:

(1) To obtain the solution at time $t+\Delta t$, we start with the initial solution guess $\underline{d}_{s}^{-1}=\underline{d}_{s}^{0}={ }^{t} \underline{d}_{s}$ and $\tau_{f}^{0}={ }^{t} \tau_{f} \cdot \underline{d}_{s}$ represents solid displacement, and $\tau_{f}$ is fluid stress. The underlining denotes that the values are defined only on the fluid-structure interfaces.

(2) Solve the fluid solution vector $X_{f}^{k}$ from the fluid equation. $k$ is the number of iteration $(k=1,2$, etc.). $\lambda_{d}$ is the displacement relaxation factor:

$$
F_{f}\left[X_{f}^{k}, \lambda_{d} \underline{d}_{s}^{k-1}+\left(1-\lambda_{d}\right) \underline{d}_{s}^{k-2}\right]=0
$$

(3) Solve the solid solution vector $X_{s}^{k}$ from the structural equation:

$$
F_{s}\left[X_{s}^{k}, \lambda_{\tau} \underline{\tau} f^{k}+\left(1-\lambda_{\tau}\right) \underline{\tau}_{f}^{k-1}\right]=0
$$

(4) The fluid nodal displacements $d_{f}^{k}$ are computed with the prescribed boundary conditions:

$$
\underline{d}_{f}^{k}=\lambda_{d} \underline{d}_{s}^{k}+\left(1-\lambda_{d}\right) \underline{d}_{s}^{k-1}
$$

(5) Both displacement and stress convergence conditions are checked according to stress and the displacement criteria. If the iteration has not converged yet, the program goes back to step 2 and continues for the next iteration unless a maximum number of FSI iterations have been reached.

Both kinematic condition and the dynamic condition are applied to the fluid-solid coupling interface to obtain compatible traction and displacement [26]. Corresponding equations are expressed as follows:

$$
\begin{aligned}
\underline{d}_{f} & =\underline{d}_{s}, \\
n \cdot \underline{\tau}_{f} & =n \cdot \underline{\tau}_{s},
\end{aligned}
$$

where $\underline{d}_{f}$ and $\underline{d}_{s}$ are the fluid and solid displacements, respectively, and $\underline{\tau}_{f}$ and $\underline{\tau}_{s}$ are the fluid and solid stresses, respectively.

Figure 6 displays the boundary conditions applied in the current simulation. Based on Arbitrary Lagrangian-Eulerian (ALE) theory, sliding mesh boundary conditions are employed to connect rotating domains and to ensure the compatibility, continuity, and completeness conditions along the nonconforming interfaces. Sliding mesh I is placed between the rotating region of the fluid around runner and the static mesh of the fluid around the guide vane. Sliding mesh II is placed between the rotating runner region and the draft tube. FSI boundary condition is imposed on the interfaces between the fluid and solid model, including the interfaces between the entire water flow and the surrounding concrete, water and the rotating blades, water and the stay vanes, water and the guide vanes. The SIMPLE algorithm is adopted to solve the pressure and velocity coupling. The upwind scheme is applied to the governing equations of the fluid part to ensure the stability of the calculation process. In equation, residual mass convergence criteria are adopted, while, in variable, residual both velocity and pressure convergence criteria are employed. The equations are required to be converged at every time step after iteration. The time step is 0.002222 seconds, corresponding to $1^{\circ}$ of the runner rotation. The total calculation time is set as 8 seconds to acquire stable simulation results.

\section{Research on the Vortex Characteristics and the Pressure Pulsation Regularities}

3.1. Transient Fluid Behavior. Figure 7 depicts the pressure and streamlines distribution in the whole flow passage. Pressure gradually decreases along flow direction from the spiral case, to stay vane, guide vane, and runner, with strong regularity of pressure distribution in the entire fluid domain. The maximum pressure magnitude in the spiral case is approximately $0.9 \mathrm{MPa}$. As the water flows into the runner, the pressure sharply decreases due to the shrinking cross section and increasing flow acceleration. In the draft tube, most of the kinetic energy is converted into static pressure thereby delimiting outlet velocity to minimal. It can be inferred from Figure 7 that there is no flow collision in the 


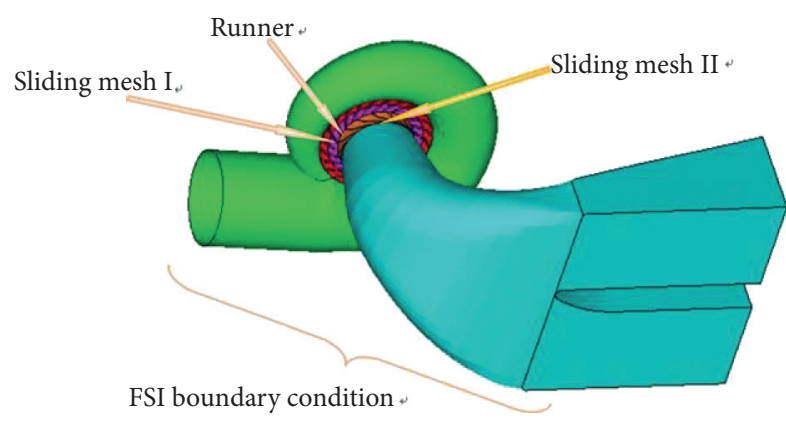

FIGURE 6: Boundary condition diagram.

flow passage, which confirms the stable operation of the turbine.

Analysis of flow structure in the draft tube plays an important role in pressure pulsation prediction and turbine performance evaluation. The Q-criterion is considered as a criterion with explicit physical meaning and hereon is adopted to analyze the vortex structure in the flow domain. $Q$-criterion represents the relative strength of the rotation to axial deformation and locates regions where rotation dominates strain in the flow [27]. The equation form for the incompressible flow is given as follows [28]:

$$
\mathrm{Q}=\frac{1}{2}\left(\|\Omega\|^{2}-\|S\|^{2}\right) \text {. }
$$

where $Q$ is the second invariant of $\nabla u, S$ and $\Omega$ denote the symmetric and antisymmetric parts of $\nabla u$, respectively, and $\|\cdot\|$ is the Euclidean matrix norm. A coherent vortex is defined as a region where $Q>0$.

Figure 8 shows typical vortices in the flow behind runner calculated by $Q$-criterion and colored by static pressure. A short and straight vortex is obtained in the straight cone of the draft tube, and its shape and strength remain steady with runner rotation. It is well known that the shape of the vortex rope is usually related to the opening of the vane and greatly affects the strength of the pressure pulsation. In the current research, the normal operating process is investigated when the turbine operates at the rated head with the rated output, and the generated vortex rope is short and straight, which predicts lower pressure pulsation caused by the vortex in the draft tube during normal operation.

3.2. Comparison with the Measurement Data. In order to evaluate the pressure pulsation level in the flow-induced vibrations, four measuring points F01 F04 are chosen and placed at head cover, vaneless region, bottom ring, and draft tube, respectively, as shown in Figure 9. Figure 10 displays the time-history curves for pressure pulsation recorded during the numerical calculation. Note that the pressure pulsation is the realistic value that contains a constant value during the calculation process. The pressure coefficient may be more effective in presenting the pressure pulsations by excluding the constant pressure. But we want to show the actual pressure pulsation values, so the coefficient is not adopted here. Periodical behavior is observed at four positions with the occurrence of fifteen wave peaks and wave troughs during one blade rotational period of $0.8 \mathrm{~s}$, which is consistent with fifteen blades rotation rule of the turbine and demonstrates evident contribution of rotor-stator-interaction to the pulsation. The wave shape of the pressure pulsation at the draft tube is largely different from that at the other three positions, which could be ascribed to the measuring positions relative to the runner. The approximate fluctuation amplitudes of the pressure at head cover, vaneless region, bottom ring, and $0.3 \mathrm{D}$ of the draft tube are $2.5 \mathrm{kPa}, 8 \mathrm{kPa}, 4 \mathrm{kPa}$, and $8 \mathrm{kPa}$, respectively.

The peak calculation of the pressure pulsation and the swing for the in-site data is $97 \%$ confidence fusion 8 -cycle averaging period method. To compare with the in-site data, the intensity of the simulated pressure oscillations is measured by the parameter of peak-to-peak value $\Delta H / H$, where $H$ is the rated head in the current operation point, and $\Delta H$ is the peak-to-peak value calculated by $97 \%$ confidence. Based on the probability and statistics method, $3 \%$ of untrusted data is eliminated from the original data to calculate the difference between the minimum and maximum value. Table 2 shows the comparison of the calculated and in-site peak to peak values of the relative pressure pulsations with 97\% confidence for different measuring points. The in-site data could be referred to report [29]. The computed $\Delta H / H$ at head cover and bottom ring are slightly lower than in-site data, while the relative pressure pulsation is slightly overestimated at the vaneless region. Improved results are expected if LES turbulence model is applied together with finer grids. As a whole, amplitudes of the pulsations agree well with the in-site data, which represents the potential of the method to predict turbine performance and thus provides a reliable basis for the simulation of transient responses of the structure.

\section{Research on Vibration Regularities of the Hydropower House}

In order to characterize and understand the vibration features of the hydropower structure, several important indexes including dynamic displacement, velocity, and acceleration are adopted to study and evaluate the structural vibration.

Figure 11 distributes the extreme value of the dynamic displacement of the hydropower house. In order to understand vibration values inside the powerhouse, Figure 11(b) displays the section diagram. Here is more illustration about the absence of upper structure like sidewall and tailwater platform. Distribution of their vibration values could make unclear the regularity of the main structural components due to their relatively higher vibration level. Thus, the analysis of them is not included in the current research. As shown in Figure 11, dynamic displacement decreases gradually from a higher elevation and the unit center to downstream structures. It is reasonable because the vibration of the concrete structure surrounding units is directly affected by the fluctuating fluid pressure produced during the unit operation. The superstructure like generator layer has a relatively higher vibration level due to the displacement amplification effect. The extreme dynamic displacement appears at the junction of the generator floor and 


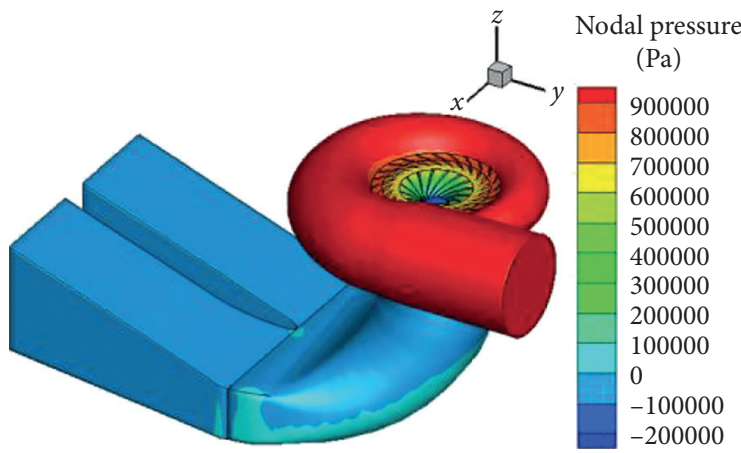

(a)

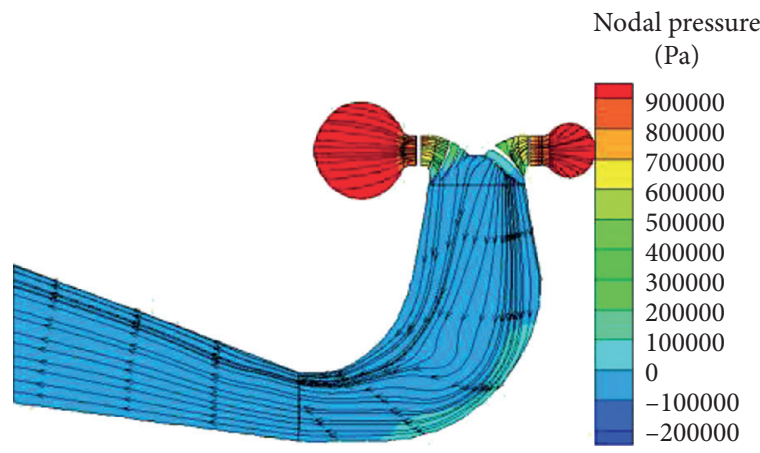

(b)

FIGURE 7: Instantaneous nodal pressure and streamlines distribution in the whole flow domain. (a) Nodal pressure in the whole domain. (b) Nodal pressure and streamlines in the meridian plane.

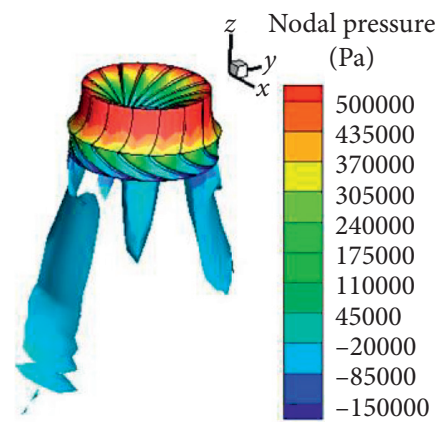

FIgURE 8: Shape of vortex rope in the draft tube.

the upstream side wall, with a value of $16.55 \mu \mathrm{m}$. The value does not exceed the limit of $0.200 \mathrm{~mm}$ in relevant vibration specifications and satisfies displacement requirements.

It is helpful to further understand the transient responses by analyzing the time-varying dynamic displacement. Extreme dynamic displacement point $A$ of the lower block structure in Figure 11 is selected to conduct time and frequency analysis, and the result is demonstrated in Figure 12. The dynamic displacement fluctuates between $0.192 \mathrm{~mm}$ to $0.200 \mathrm{~mm}$ periodically. Based on the Fast Fourier Transform (FFT) algorithm, frequency domain analysis is conducted and shown in Figure 12(b). Here, we define $f_{n}$ as the blade rotation frequency; the value of $f_{n}$ is $1.25 \mathrm{~Hz}$. From Figure 12(b), we can see that the first dominant frequency is $30 f_{n}$, two times the blades passing frequency $15 f_{n}$, so the fluctuation of the dynamic displacement at the extreme point is mainly affected by blades passing frequency.

Figure 13 is the distribution of extreme vibration velocity of hydropower house in three-dimensional and section view. The dynamic velocity represents a strong regularity with dynamic velocity diminishing from the unit center. The diffuse section of the draft tube, which is far away from the unit, owns a relatively lower vibration velocity level and the value is about $0.2 \mathrm{~mm} / \mathrm{s}$. Relatively drastic vibration velocity occurs at the entrance of the straight cone section of the draft tube, which is approximately below a 130-degree envelope angle of the spiral case. The extreme value of the vibration velocity is $1.13 \mathrm{~mm} / \mathrm{s}$, which conforms to the restrictive requirement of $5.00 \mathrm{~mm} / \mathrm{s}$ in relevant vibration specifications.

Figure 14 reveals the time history and frequency spectrum of the extreme velocity point $B$. The vibration velocity of the extreme point fluctuates between 0 and $1.20 \mathrm{~mm} / \mathrm{s}$ periodically, and the wave is denser compared to timevarying dynamic displacement. Figure 14(b) shows the frequency domain graph of the extreme velocity point $B$. More frequency components are detected largely times the blades passing frequency $15 f_{n}$. The first dominant frequency is 60 times rotational frequency $f_{n}$, which indicates the velocity vibration is greatly affected by the rotation of runner.

The maximum acceleration distribution of the hydropower house is shown in Figure 15. Although it is easily detected that the distribution regularity of acceleration extreme value is similar to that of vibration velocity, we want to present not only the location of the maximum variable in the graph but also the general distribution of the variable values. Significant vibration acceleration appears at the entrance of the straight cone section of the draft tube, and the value gradually decreases to concrete structures around. The vibration level in most of the areas is less than $0.20 \mathrm{~m} / \mathrm{s}^{2}$. In the lower block structure, especially the diffuser of the draft tube, the vibration acceleration is at a much lower level. In order to see the distribution of vibration acceleration inside the 


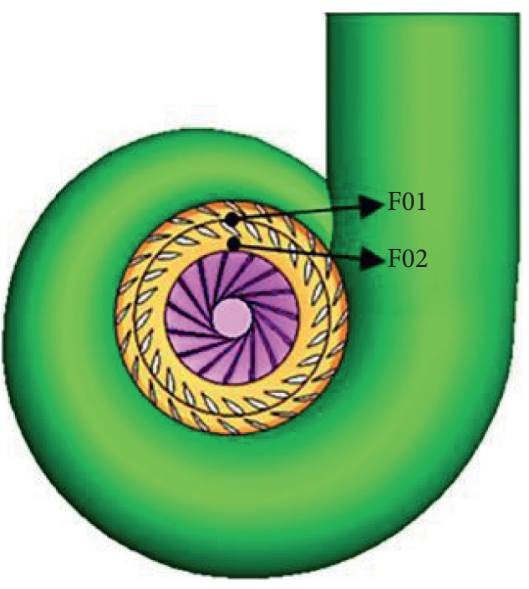

(a)

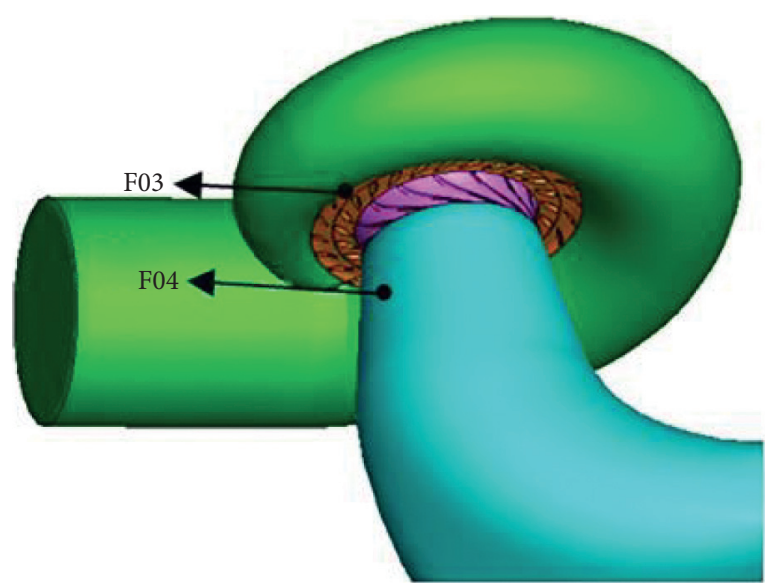

(b)

FIGURE 9: Pressure monitoring points in the fluid flow channels. (a) Location for measuring points F01 and F02. (b) Location for measuring points F03 and F04.

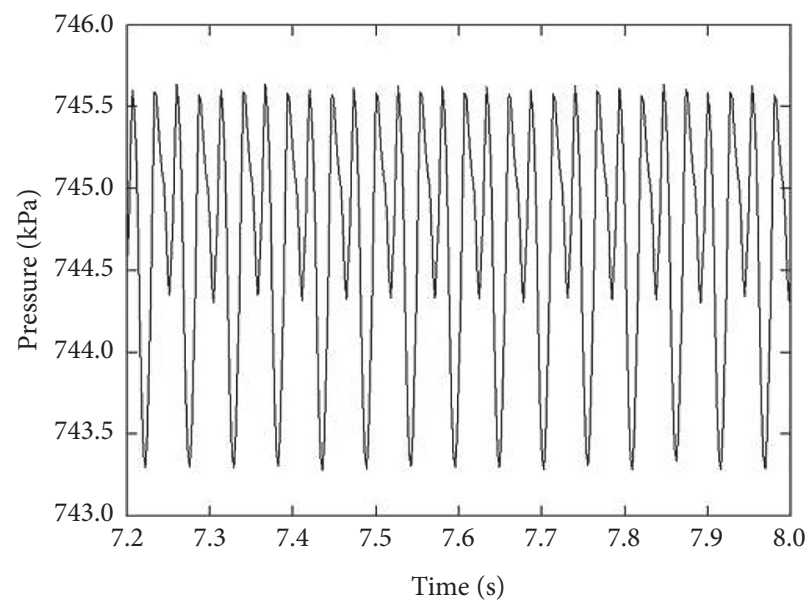

(a)

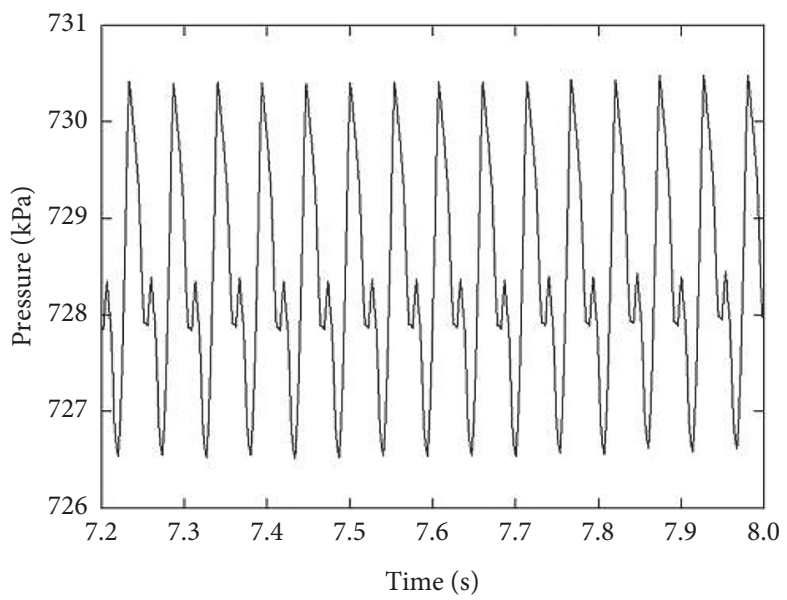

(c)

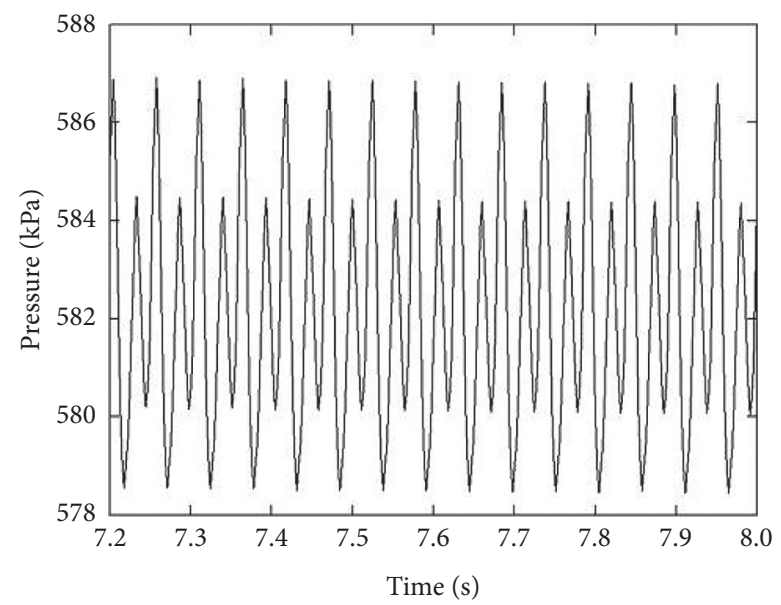

(b)

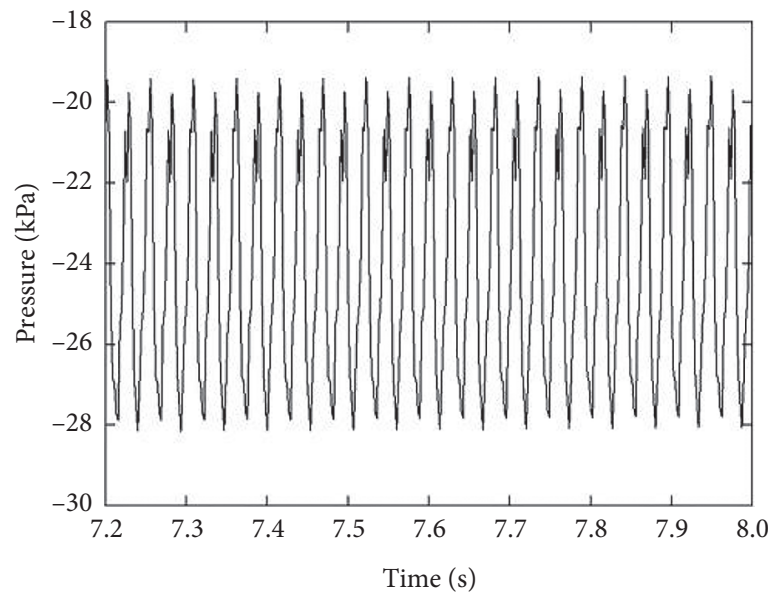

(d)

FiguRE 10: Numerical simulation results of time history of pressure pulsation at different positions in the fluid domain. (a) Measuring point F01 at head cover. (b) Measuring point F02 at the vaneless region. (c) Measuring point F03 at the bottom ring. (d) Measuring point F04 at $0.3 \mathrm{D}$ of the draft tube. 
TABLE 2: $\Delta H / H$ with $97 \%$ confidence at different positions for simulation and in-site data (Table 2 is reproduced from Shuo Wang et al., 2019.).

\begin{tabular}{lccc}
\hline Location & \multicolumn{4}{c}{$\Delta H / H$ with $97 \%$ degree of confidence (\%) } & Error (\%) \\
& Simulation results & In-site data & 16 \\
Head cover & 0.26 & 0.31 & 4 \\
Vaneless region & 0.85 & 0.82 & 2.41 \\
Bottom ring & 0.40 & 2 & 4 \\
\hline
\end{tabular}

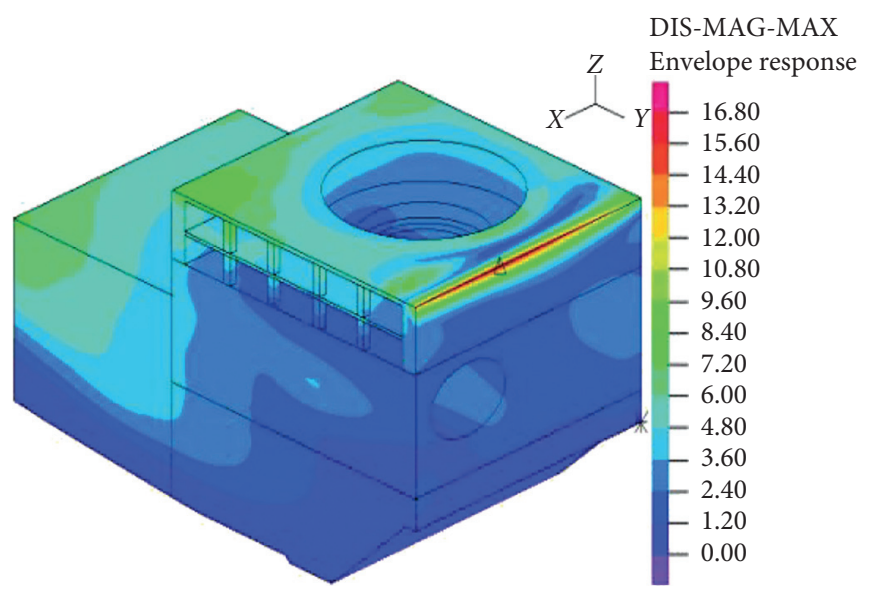

(a)

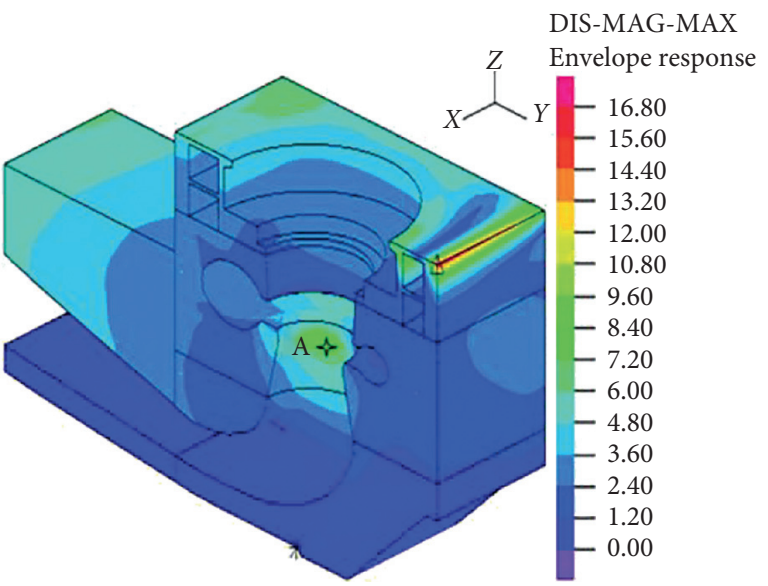

(b)

FIGURE 11: Extreme dynamic displacement distribution of the concrete structure $(\mu \mathrm{m})$. (a) In three-dimensional view. (b) In section view.

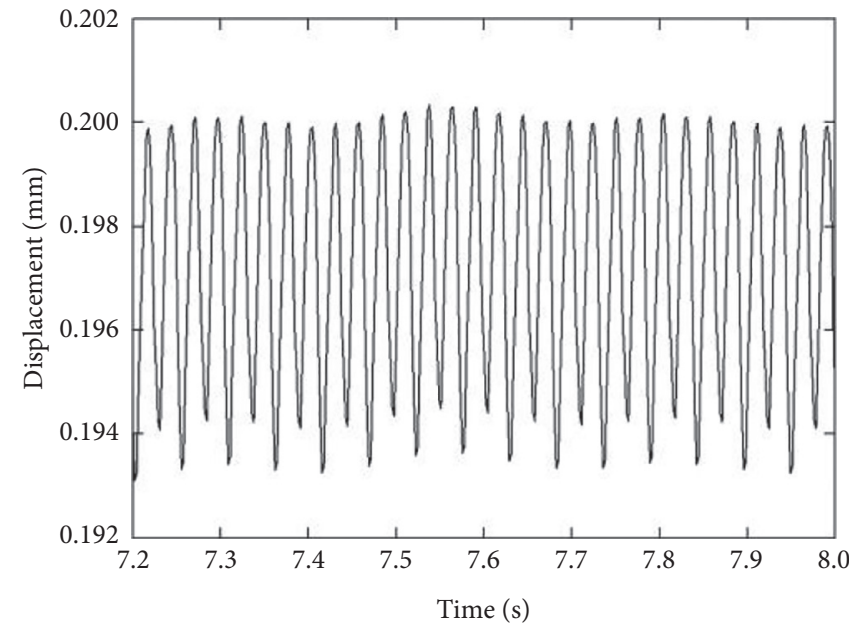

(a)

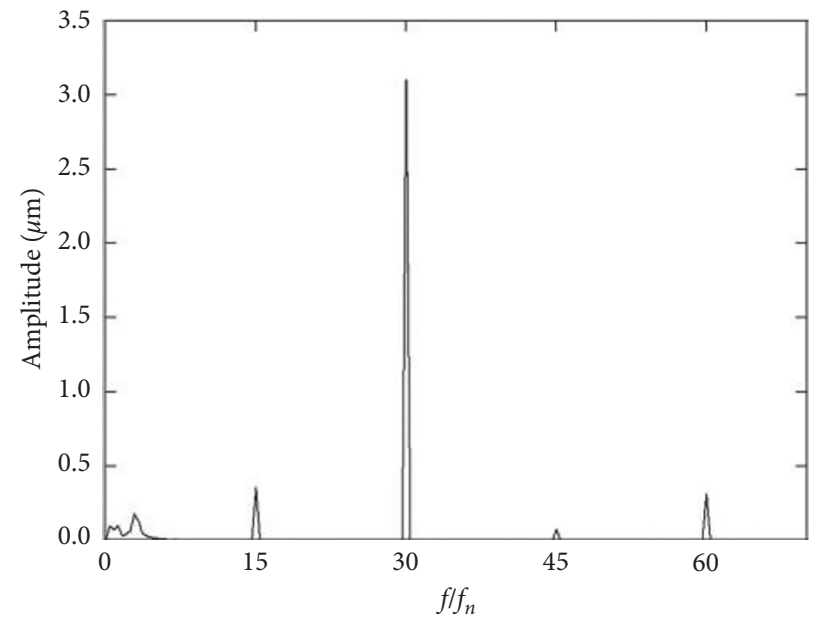

(b)

Figure 12: Displacement fluctuation analysis of the extreme dynamic displacement point (A). (a) Time-domain graph. (b) Frequencydomain graph.

house, the section diagram is provided in Figure 15(b). The position of the extreme acceleration point of the structure is close to that of the extreme velocity point. Both of them are located at about a 130-degree envelope angle of the spiral case in the entrance concrete of the straight cone section. The calculated acceleration values are lower than the criterion value of $1.00 \mathrm{~m} / \mathrm{s}^{2}$ in the control criterion of hydropower house vibration.
Extreme acceleration point $C$ is selected and the corresponding time-varying and frequency spectrum is shown in Figure 16. Figure 16(a) describes the variation range and regularity of the extreme vibration acceleration point during one blade rotational period. Acceleration fluctuates between 0 and $0.45 \mathrm{~m} / \mathrm{s}^{2}$ steadily and periodically. Figure $16(\mathrm{~b})$ reports the main frequency components based on the FFT algorithm. The first dominant frequency of vibration 


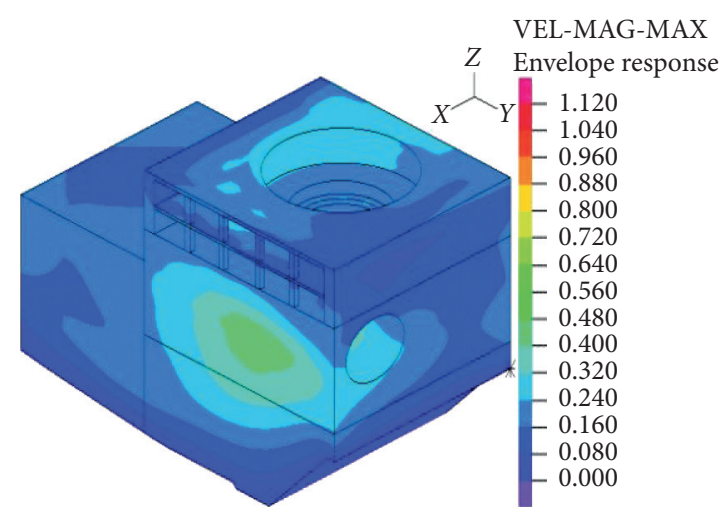

(a)

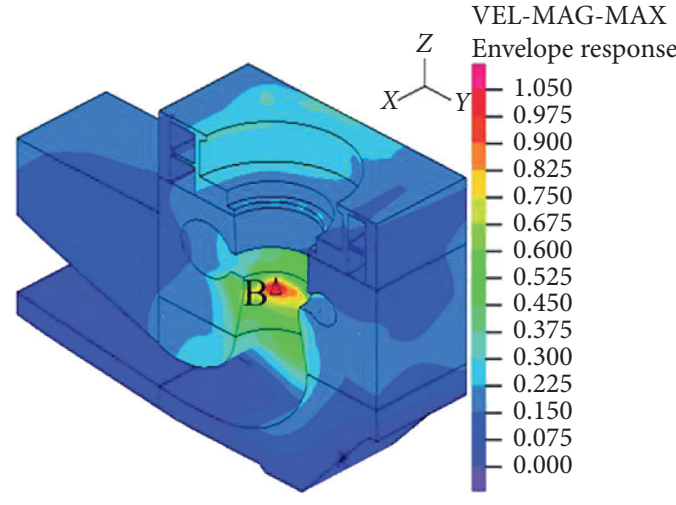

(b)

FIgURE 13: Extreme velocity distribution of the concrete structure (mm/s). (a) In three-dimensional view. (b) In section view.

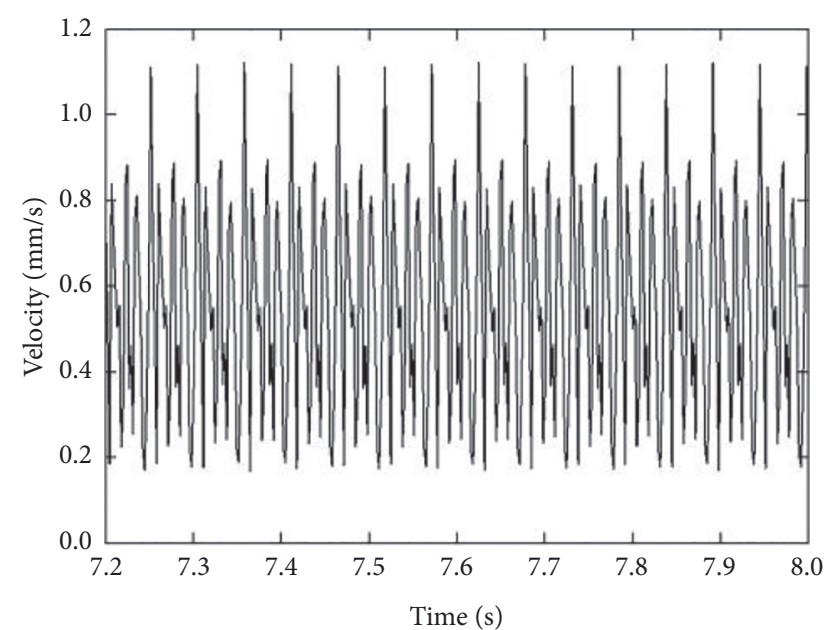

(a)

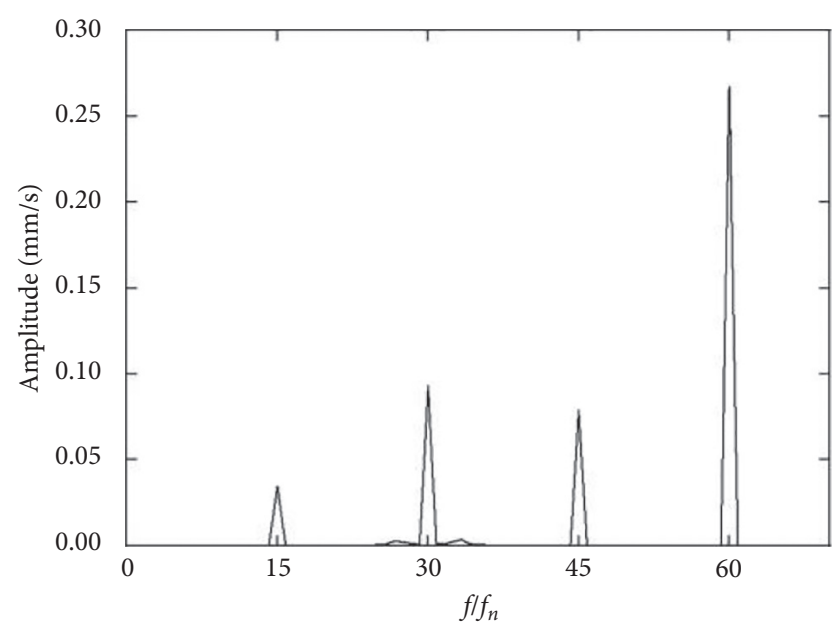

(b)

FIGURE 14: Velocity fluctuation analysis of the extreme velocity point (B). (a) Time-domain graph. (b) Frequency-domain graph.

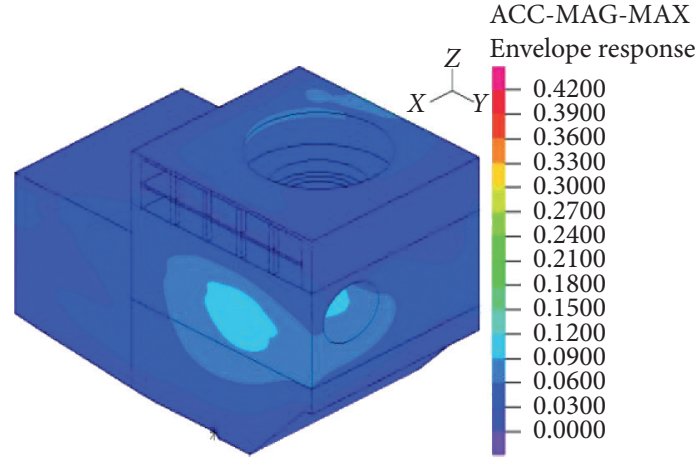

(a)

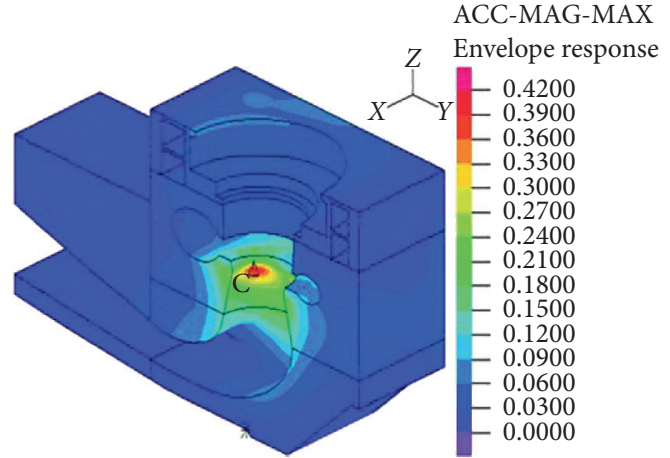

(b)

FIGURE 15: Extreme acceleration distribution of the concrete structure $\left(\mathrm{m} / \mathrm{s}^{2}\right)$. (a) In three-dimensional view. (b) In section view. 


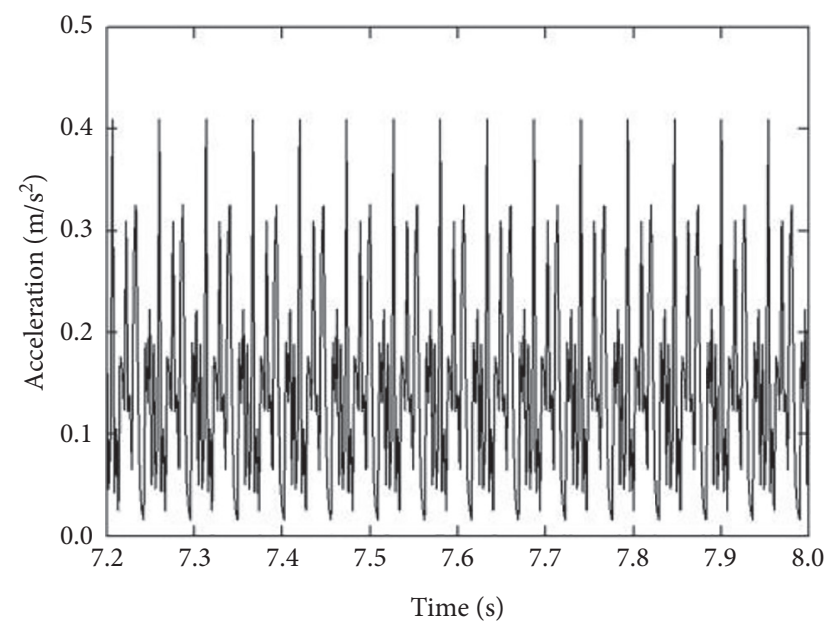

(a)

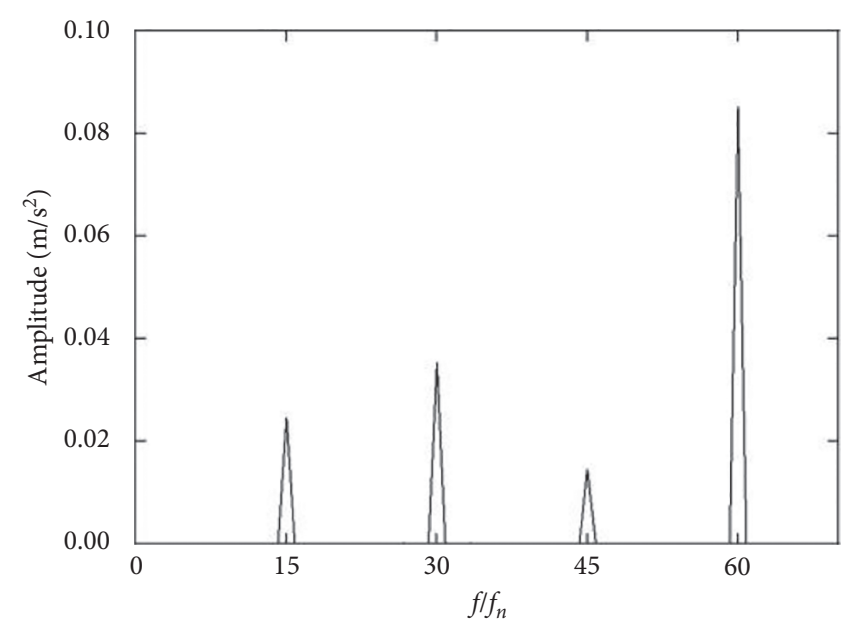

(b)

Figure 16: Acceleration fluctuation analysis of the extreme acceleration point $(C)$. (a) Time-domain graph. (b) Frequency-domain graph.

acceleration is $60 f_{n}$. Other main frequencies are largely multiple times the blade passing frequency $15 f_{n}$. The blades rotation is the major contributor to structural vibration. The above analysis shows that the vibration caused by the rotation of the turbine blade is the main vibration source of the hydropower house.

\section{Conclusion}

This paper combined CFD theory and CSD theory, discussed the bidirectional FSI simulation analysis theory and calculation method for the large hydropower houses, and explored the general law of the vortex-induced vibration. Using the famous structural analysis software ADINA, a 3D FSI finite element model of the fluid-hydraulic mechanics-concrete structure of the Xiangjiaba hydropower house was established. The vortex-induced vibration mechanism and coupled vibration law were explored during transient unit operation. The following main conclusions were obtained:

(1) The time-varying pressure pulsations at typical positions of head cover, vaneless region, bottom ring, and draft tube were analyzed and verified with the insite measuring data. The results showed that the pressure distribution was reasonable in the flow passage, and the streamlines were smooth in the entire flow passage under the design condition. The short and straight shape of the vortex generated behind the runner indicated the low intensity of pressure pulsations. A comparison of the in-site data with the calculated values showed that the calculated results could satisfactorily describe the behavior of the vortex features and pressure pulsations in the fluid domain during design operation, which laid the foundation for turbine's performance prediction and the structural vibration evaluation.

(2) The responses of the vibration displacement, vibration velocity, and vibration acceleration of the hydropower house were studied, and the vibration law of the hydropower house during the unit operation was discussed. The results showed that the extreme values of the vibration displacement of the hydropower house occurred on the generator floor. Both the extreme values of the vibration velocity and vibration acceleration appeared at the entrance of the straight cone of the draft tube. The extreme values were lower than the criterion value, which satisfied the vibration control requirements. The dominant frequencies of the extreme point of vibration displacement, vibration velocity, and vibration acceleration in lower block concrete structure were multiple of blades passing frequency, indicating the blades rotation was the main vibration source during operation.

In the current paper, our main focus is on the vibration features of the hydropower house in design operation. The vibration features of operation of the Francis turbines at offdesign conditions will be our further focus.

\section{Data Availability}

Some or all data, models, or codes generated or used during the study are available from the corresponding author upon request.

\section{Conflicts of Interest}

The authors declare that there are no conflicts of interest regarding the publication of this paper.

\section{Acknowledgments}

This work was financially supported by the National Key Research and Development Plan of China (Grant no. 2017YFC0404903) and the Fundamental Research Funds for the Central Universities (Grant no. 2016B41014). 


\section{References}

[1] Z. Liming, L. Yongyao, W. Zhengwei, L. Xin, and X. Yexiang, "A review on the large tilting pad thrust bearings in the hydropower units," Renewable and Sustainable Energy Reviews, vol. 69, pp. 1182-1198, 2017.

[2] C. Trivedi and M. J. Cervantes, "Fluid-structure interactions in francis turbines: a perspective review," Renewable and Sustainable Energy Reviews, vol. 68, no. 1, pp. 87-101, 2017.

[3] C. Trivedi, J. M. Cervantes, and G. O. Dahlhaug, "Experimental and numerical studies of a high-head francis turbine: a review of the francis-99 test case," Energies, vol. 9, no. 2, p. 74, 2016.

[4] D. Li, Y. Sun, Z. Zuo, S. Liu, H. Wang, and Z. Li, “Analysis of pressure fluctuations in a prototype pump-turbine with different numbers of runner blades in turbine mode," Energies, vol. 11, no. 6, p. 1474, 2018.

[5] A. Kc, Y. H. Lee, and B. Thapa, "CFD study on prediction of vortex shedding in draft tube of francis turbine and vortex control techniques," Renewable Energy, vol. 86, pp. 14061421, 2016.

[6] A. V. Minakov, D. V. Platonov, A. A. Dekterev, A. V. Sentyabov, and A. V. Zakharov, "The analysis of unsteady flow structure and low frequency pressure pulsations in the high-head francis turbines," International Journal of Heat and Fluid Flow, vol. 53, pp. 183-194, 2015.

[7] X.-Z. Wei, W.-T. Su, X.-B. Li, F.-C. Li, and L. Guo, "Effect of blade perforation on francis hydro-turbine cavitation characteristics," Journal of Hydraulic Research, vol. 52, no. 3, pp. 412-420, 2014.

[8] C. Trivedi, M. Cervantes, and B. Gandhi, "Investigation of a high head francis turbine at runaway operating conditions," Energies, vol. 9, no. 3, p. 149, 2016.

[9] I. Chalghoum, S. Elaoud, M. Akrout, and E. H. Taieb, "Transient behavior of a centrifugal pump during starting period," Applied Acoustics, vol. 109, pp. 82-89, 2016.

[10] L. Deyou, W. Hongjie, X. Gaoming, G. Ruzhi, W. Xianzhu, and L. Zhansheng, "Unsteady simulation and analysis for hump characteristics of a pump turbine model," Renewable Energy, vol. 77, pp. 32-42, 2015.

[11] J. Zhang, H. Wu, J. Liu, and C. Shi, "Underground powerhouse vibration responses to different frequency domain pressure fluctuations in hongping pumped storage power station," China Rural Water \& Hydropower, no. 6, pp. 155159, 2016.

[12] H. Wang, B. Bai, and K. Li, "Research on vibration propagation regular of adjacent unit-blocks for hydropower house," Journal of Water Resources \& Water Engineering, vol. 27, no. 1, pp. 141-146, 2016.

[13] C.-H. Zhang and Y.-L. Zhang, "Nonlinear dynamic analysis of the three gorge project powerhouse excited by pressure fluctuation," Journal of Zhejiang University-Science A., vol. 10, no. 9, pp. 1231-1240, 2009.

[14] R. Negru, S. Muntean, L. Marsavina, R. Susan-Resiga, and N. Pasca, "Computation of stress distribution in a francis turbine runner induced by fluid flow," Computational Materials Science, vol. 64, pp. 253-259, 2012.

[15] R. A. Saeed, A. N. Galybin, and V. Popov, "Modelling of flowinduced stresses in a Francis turbine runner," Advances in Engineering Software, vol. 41, no. 12, pp. 1245-1255, 2010.

[16] R. Xiao, Z. Wang, and Y. Luo, "Dynamic stresses in a francis turbine runner based on fluid-structure interaction analysis," Tsinghua Science and Technology, vol. 13, no. 5, pp. 587-592, 2008.
[17] A. Luna-Ramírez, A. Campos-Amezcua, O. Dorantes-Gómez, Z. Mazur-Czerwiec, and R. Muñoz-Quezada, "Failure analysis of runner blades in a francis hydraulic turbine -case study," Engineering Failure Analysis, vol. 59, pp. 314-325, 2016.

[18] F. Dompierre and M. Sabourin, "Determination of turbine runner dynamic behaviour under operating condition by a two-way staggered fluid-structureinteraction method," in Proceedings of the IOP Conference Series: Earth and Environmental Science, vol. 12, no. 1, p. 9, September 2010, Article ID 012085.

[19] H. Schmucker, F. Flemming, and S. Coulson, "Two-way coupled fluid structure interaction simulation of a propeller turbine," in Proceedings of the Iop Conference Series Earth \& Environmental Science, vol. 12, pp. 793-798, Politehnica' University of Timişoara, Timişoara, Romania, September 2010.

[20] Z. Zhang, H. Wu, Z. Qian, K. Su, and C. Shi, "Flow-induced vibration analysis of hydropower house based on data transfer model for fluid-structure coupling interface," Advanced Engineering Sciences, vol. 49, no. S2, pp. 8-15, 2017, in Chinese.

[21] A. Gavrilov, A. Dekterev, A. Minakov, D. Platonov, and A. Sentyabov, "Application of hybrid methods to calculations of vortex precession in swirling flows," vol. 117, pp. 449-459, in Proceedings of the Sixth Symposium on Progress in Hybrid RANS-LES Methods, vol. 117, pp. 449-459, Springer, Strasbourg, France, September 2012.

[22] P. Spalart and S. Allmaras, "A one-equation turbulence model for aerodynamic flows," in Proceedings of the 30th Aerospace Sciences Meeting and Exhibit, p. 439, USA, April 1992.

[23] Y. Guo and C. Kato, "Investigation of the performance of DES-SA model in several turbulent flows," Seisan Kenkyu, vol. 60, no. 1, pp. 75-80, 2008.

[24] S. Tu, A. Shahrouz, P. Reena, and W. Marvin, "An implementation of the Spalart-Allmaras DES model in an implicit unstructured hybrid finite volume/element solver for incompressible turbulent flow," International Journal for $\mathrm{Nu}$ merical Methods in Fluids, vol. 59, no. 9, pp. 1051-1062, 2010.

[25] A. Pálfalvi, "Efficient solution of a vibration equation involving fractional derivatives," International Journal of Nonlinear Mechanics, vol. 45, no. 2, pp. 169-175, 2010.

[26] S. Wei and L. Zhang, "Vibration analysis of hydropower house based on fluid-structure coupling numerical method," Water Science and Engineering, vol. 3, no. 1, pp. 75-84, 2010.

[27] M. A. Green, C. W. Rowley, and G. Haller, "Detection of Lagrangian coherent structures in three-dimensional turbulence," Journal of Fluid Mechanics, vol. 572, no. 572, pp. 111-120, 2007.

[28] J. Jeong and F. Hussain, "On the identification of a vortex," Journal of Fluid Mechanics, vol. 285, no. 1, pp. 69-94, 1995.

[29] Y. Yin, B. Peng, H. Si, J. Wang, and C. Cao, "Test report on unit stability and energy features of Xiangiiaba," Hydropower Station1F (HEC) (Upstream Water Level of $370 \mathrm{~m}$ to $380 \mathrm{~m}$ )in Chinese, 2014. 